\title{
A Prospective Study on the Epidemiology of Onychomycosis in Tertiary Care Hospital
}

\author{
Vinay Hajare $^{1 *}$, G.P. Aaftab ${ }^{2}$ and Abdul Hadi Waseem ${ }^{3}$ \\ Ram Mandir, Shahabazar, Gulbarga 585101, India \\ *Corresponding author
}

\section{A B S T R A C T}

Fungal infection of nails or onychomycosis is non-life threatening disease commonly caused by dermatophytes. The infection is also caused by non dermatophytes like yeasts and non dermatophytic moulds. There are various factors which play an important role in causation of onychomycosis. These predisposing factors are aging, fall in the immune status, diabetes, immunosuppressive therapy for cancer and organ transplantation, HIV, long term antibiotics, occlusive footwear, immune deficiency diseases and occupations involving continuous contact with water, for instance swimmers, fishermen, clothes and dish washers. Climatic conditions also play an important role in the causation of onychomycosis. The present study was carried out in a tertiary care hospital for a period of

\section{Keywords}

Onychomycosis, Dermatophytes, Trichophyton.

\section{Article Info}

Accepted:

20 July 2018

Available Online:

10 August 2018

8 months. The aim of the study was to determine various predisposing factors and causative agents of onychomycosis. The sample was placed in a sterile petridish and transported to microbiology laboratory. The sample was then divided into two parts, one for direct microscopy under high power objective using 20-25\% $\mathrm{KOH}$ and the other part for culture on Sabouraud's dextrose agar (SDA) with cyclohexamide. The cultures were kept at $25^{\circ} \mathrm{C}$ and $37^{\circ} \mathrm{C}$ for up to six weeks. Confirmation of the organism was done based on morphology of fungus in LPCB (Lactose phenol cotton blue) mount, culture of fungus on SDA and slide culture. Among the 68 patients selected based on clinical presentation, 26 yielded fungal pathogens in culture. A total of $15(57.6 \%)$ isolates were dermatophytes and $11(42.3 \%)$ were non dermatophytes. Among the dermatophytes, 7 (26.9\%) cases yielded Trichophyton which was the most commonly isolated fungus followed by Microsporum 5 (19.2\%), Epidermophyton 3 (11.5\%). Among the non dermatophytes, candida was isolated from $3(11.5 \%)$ cases, Aspergillus was isolated from $2(7.6 \%)$, Pyrenochaeta from 2 (7.6\%) cases, Curvularia from 2 (7.6\%) cases and only $1(3.8 \%)$ case yielded Fusarium. It was seen that males were more prone to onychomycosis compared to females. Incidence of toe nail onychomycosis was higher compared to finger nail onychomycosis. This study suggests that the isolation of the organism with culture is very important as it will aid the clinician to rule out bacterial causes and choose appropriate antifungal therapy.

\section{Introduction}

Fungal infection of nails or onychomycosis is non-life threatening disease commonly caused by dermatophytes. The infection is also caused by non dermatophytes like yeasts and non dermatophytic moulds. There are various factors which play an important role in 
causation of onychomycosis. These predisposing factors are, aging, fall in the immune status, diabetes, immunosuppressive therapy for cancer and organ transplantation, HIV, long term antibiotics, wearing of occlusive footwear, immune deficiency diseases and occupations involving continuous contact with water, for instance swimmers, fishermen, clothes and dish washers Kaur et al., (2007).Climatic conditions also favour onychomycosis. It was concluded that the prevalence of onychomycosis was low in tropical countries $(3.8 \%)$ than in subtropical and temperate zones (18\%) (Bramono et al., 2001).

Although onychomycosis is merely a cosmetic problem, it can cause a more serious health problem in HIV infected patients. Onychomycosis in non immunocompromised patients can cause negative effects like social and emotional embarrassment, nonwillingness to let their hands and feet to be seen and patients may fear that they might transmit the infection to their family members, relatives and co-workers. Differential diagnosis to onychomycosis infection includes psoriasis, lichen planus, onychogryphosis and nail trauma. Onychomycosis represents upto 20\% of nail disorders (Charif et al., 1997; Bronson et al., 1983). The prolonged therapy with its adverse effects may discourage the patients.

The dermatophyte Trichophyton rubrum is the major cause of onychomycosis (Charif et al., 1997). The second most commonly isolated fungal pathogen from onychomycosis patients is the dermatophyte Trichophyton tonsurans (Bronsonet et al., 1983). Other dermatophytes causing onychomycosis are Trichophyton mentagrophytes, Trichophyton megninii, Trichophyton schoenleinii, Microsporum gypseum and Epidermophyton floccosum. Non dermatophytic fungi like Fusarium oxysporum (Zaias et al., 1972), Scytalidium,
Scopulariopsis, Candida, Acremonium, Fusarium solani, Aspergillus, Arachnomyces, Pyrenochaeta unguis hominis have also been isolated from cases of onychomycosis.

\section{Classification of onychomycosis}

According to the clinical presentation and the route of invasion, onychomycosis can be classified into four types.

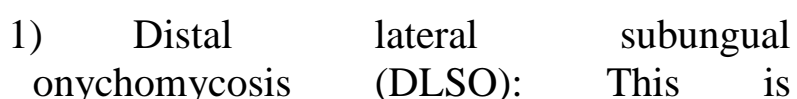
characterised by invasion of the nail bed and the underside of the nail plate, beginning at the hyponychium and leading to hyperkeratosis or onycholysis with thickening of the subungual region. The nail may appear yellowish brown in colour (Cohen et al., 1992).

2) Proximal subungual onychomycosis (PSO): also known as proximal white subungual onychomycosis is a condition where the organism invades the nail from the proximal nail fold through the cuticle area. It may present with hyperkeratosis, proximal onycholysis, leukonychia and destruction of the proximal nail plate, involving all the layers of the nail (Dompmartin et al., 1990).

3) White superficial onychomycosis (WSO): which occurs when the fungi invades the superficial layer of the nail plate leading to formation of opaque white patches on the external nail plate which coalesce and spreads as the disease progresses finally causing the nail to become rough, soft and crumbly (Cohen et al., 1992).

4) Candida infection of the nail: In this condition the organism invades the entire nail plate causing onycholysis and paronychia. Candida infection is more commonly seen in women than in men (Andre et al., 1987) and over the middle 
finger of women which frequently comes in contact with the organism residing in the vagina or intestine (Zaias et al., 1996).

The present study was carried out in a tertiary care hospital for a period of 8 months. The aim of the study was to determine various predisposing factors and causative agents of onychomycosis.

\section{Materials and Methods}

Inclusion criteria: Patients presenting with distal subungual onychomycosis, proximal subungual onychomycosis, white superficial onychomycosis, paronychia, onycholysis, hyperkeratosis, yellowish brown discoloration and dystrophy were selected for the study.

Collection and transport of Sample: The nails of the selected patients were cleansed with $80 \%$ ethanol to remove contaminating bacteria from the site. The sample was then obtained by vigorous scraping on nail bed, underside of nail plate and hyponychium. The sample was placed in a sterile petridish and transported to microbiology laboratory (Kaur et al., 2007).

Processing of the sample: The sample was then divided into two parts, one for direct microscopy under high power objective using $20 \% \mathrm{KOH}$ and the other part for culture on Sabouraud's dextrose agar (SDA) with cyclohexamide, as it prevents the growth of non dermatophytic fungi. SDA without cyclohexamide and with 5\% chloramphenicol was used to grow non dermatophytic fungi. The cultures were kept at $25^{\circ} \mathrm{C}$ and $37^{\circ} \mathrm{C}$ for up to six weeks. No growth in the media after six weeks was reported as negative (Boni et al., 1998). Confirmation of the organism was done based on morphology of fungus in LPCB (Lactose phenol cotton blue) mount done from the material obtained from the culture of fungus on SDA and slide culture (Ramudamu et al., 2018). Urease test and India Ink staining was performed to differentiate candida from Cryptococcus as Cryptococcus shows positive reaction for urease test and it is a capsulated organism unlike candida which is noncapsulated and shows negative reaction for urease test. The capsule can be demonstrated by negative staining with India ink or Nigrosin (Jagdish Chander, 2017).

\section{Results and Discussion}

Based on the clinical presentation 68 patients were selected among which fungus was isolated from $28(38.2 \%)$ cases. Male patients were more prone to onychomycosis $18(69.2 \%)$ compared to female patients $8(30.7 \%)$ (Chart 1). It was seen that $16(61.5 \%)$ isolates were from the toe nails, 7 (26.9\%) isolates were from finger nails and only 3 isolates $(11.5 \%)$ were from both toe and finger nails(Chart 2). Out of the 26 isolates, $13(50 \%)$ isolates were from Proximal subungual onychomycosis, 8 (30\%) were from distal lateral subungual onychomycosis, 2 (7.6\%) from white subungual onychomycosis and 3 $(11.5 \%)$ cases were from candida infection (Table 1). A total of $15(57.6 \%)$ isolates were dermatophytes and $11(42.3 \%)$ were other than dermatophytes (Table 2). Among the dermatophytes, Trichophyton was most commonly isolated 7 (26.9\%), followed by Microsporum 5 (19.2\%), Epidermophyton 3 (11.5\%). Among the non dermatophytes, Candida was isolated from $3(11.5 \%)$ cases, Aspergillus was isolated from 2 (7.6\%), Pyrenochaeta from 2 (7.6\%) cases, Curvularia from $2(7.6 \%)$ cases and only 1 (3.8\%) case yielded Fusarium. Comparison of various predisposing factors for Onychomycosis in males and females is depicted in Table 3. Onychomycosis is a cosmetic problem and a chronic disease which has a long duration of treatment (Fig. 1 and 2). 
Table.1 Table depicting distribution of various types of onychomycosis based on clinical presentation

\begin{tabular}{|l|l|}
\hline Clinical presentation & Isolates $(\mathrm{n}=26)$ \\
\hline Proximal subungual onychomycosis & $13(50 \%)$ \\
\hline Distal lateral subungual onychomycosis & $8(30 \%)$ \\
\hline White subungual onychomycosis & $2(7.6 \%)$ \\
\hline Candidal & $3(11.5 \%)$ \\
\hline
\end{tabular}

Table.2 Various fungal pathogens isolated from 26 onychomycosis cases

\begin{tabular}{|l|l|}
\hline Dermatophytes & \\
\hline Trichophyton & $7(26.9 \%)$ \\
\hline Microsporum & $5(19.2 \%)$ \\
\hline Epidermophyton & $3(11.5 \%)$ \\
\hline Total & $\mathbf{1 5}(\mathbf{5 7 . 6 \% )}$ \\
\hline Non dermatophytes & $3(11.5 \%)$ \\
\hline Candida & $2(7.6 \%)$ \\
\hline Aspergillus & $2(7.6 \%)$ \\
\hline Pyrenochaeta & $1(3.8 \%)$ \\
\hline Fusarium & $1(3.8 \%)$ \\
\hline Penicilium & $2(7.6 \%)$ \\
\hline Curvularia & $\mathbf{1 1}(\mathbf{4 2 . 3 \% )}$ \\
\hline Total & \\
\hline
\end{tabular}

Fig.1 Gender wise distribution of Onychomycosis

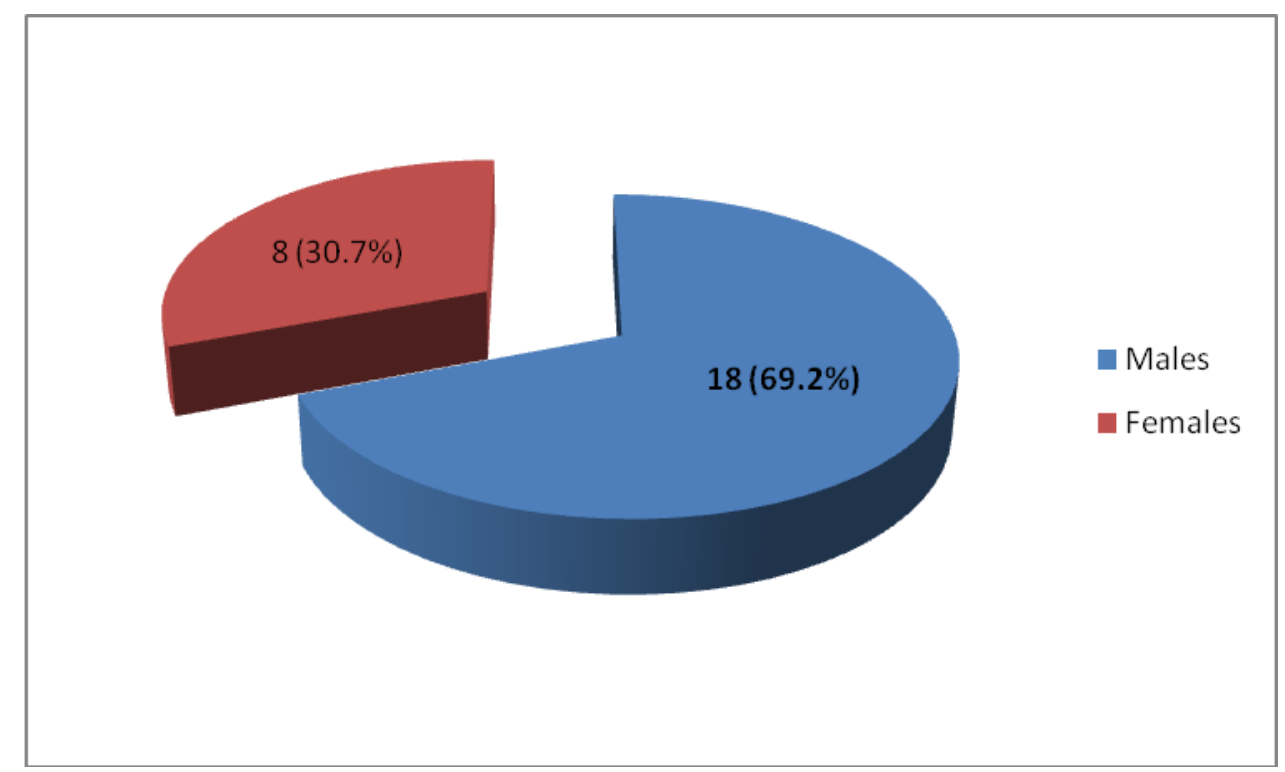


Table.3 Comparison of various predisposing factors among Onychomycosis cases $(\mathrm{n}=26)$

\begin{tabular}{|l|l|l|}
\hline Risk factors & Males & Females \\
\hline Trauma & $8(30.76 \%)$ & $4(15.38 \%)$ \\
\hline Immunocompromised & $3(11.53 \%)$ & - \\
\hline Diabetes & $1(3.84 \%)$ & $3(11.53 \%)$ \\
\hline Occupations not involving trauma & $5(19.23 \%)$ & $2(7.69 \%)$ \\
\hline
\end{tabular}

Fig.2 Fungal isolation from different sites

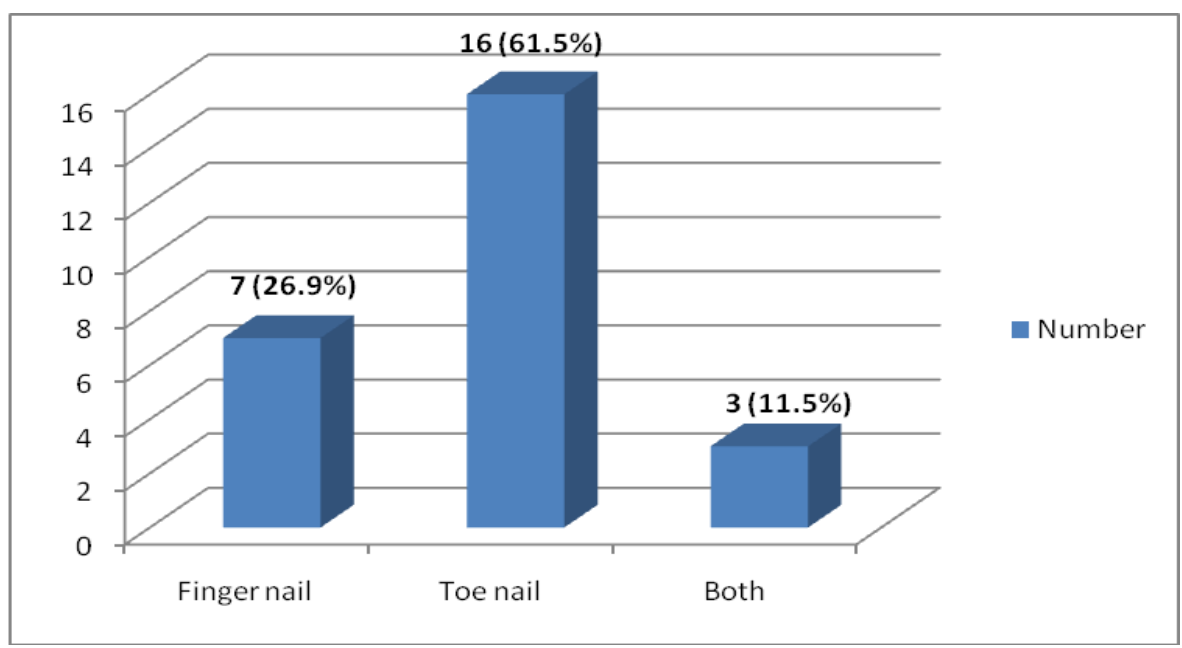

Our study showed an isolation rate of $38.2 \%$ which was low when compared to Heikkila et al., (1995), who isolated fungus from 91 (56.17\%) clinical samples among the 162 patients selected based on clinical presentation.

In the present study it was seen that males were very prone to onychomycosis compared to females which correlates with the study conducted by Sigurgeirsson et al., (2014) In our study, fungus was more commonly isolated from cases presenting with proximal subungual onychomycosis which was in contrary to study by Adekhand et al., (2015) who isolated fungus more commonly from distal lateral subungual onychomycosis. In comparison to Aditya et al., (2000), our study also showed a higher incidence of toe nail onychomycosis. Dermatophytes were the most common organisms isolated. Our results were almost similar to the findings of Gupta et al., (2000) who also showed a higher incidence of onychomycosis by dermatophytes. Among the dermatophytes, Trichophyton was most commonly isolated. Our study had similar results with Mugge et al., (2006). Verylittle is known about the risk factors for onychomycosis. Trauma is the major cause of onychomycosis accounting for $8(30.76 \%)$ in males and 4 (15.38\%) in females, followed by occupations not involving trauma such as fisher men, clothes and utensil washers, swimmers etc. Even in this group men are predominantly infected. The incidence of onychomycosis in diabetes and immunocompromised patients was less.

In conclusion, onychomycosis is a growing public health concern. Dermatophytes are the primary cause of onychomycosis when compared with non-dermatophytes. Onychomycosis occurs more commonly in men compared to women. The cause may be related to the occupations where the incidence of trauma is more like carpentry, agriculture, wood cutting, iron smith and in some instances it may 
be non-occupational like using occlusive footwear and many other such factors. Diabetes and immune compromised conditions promote onychomycosis. Isolation of the organism with culture is very important as it will aid the clinician to rule out bacterial causes and choose appropriate antifungal therapy.

\section{References}

Adekhandi S, Pal S, Sharma N, Juyal D, Sharma M, Dimri D. Incidence and epidemiology of onychomycosis in patients visiting tertiary care hospital in India. Cutis, 2015; 95(1): E20-5.

Aditya K. Gupta, Hem C. Jain, Charles W. Lynde, Paul Mac Donald, Elizabeth A. Cooper, and Richard C. Summerbell. Prevalence and epidemiology of onychomycosis in patients visiting physicians offices: A multicentre Canadian Survey of 15000 patients. J Am Acad Dermatol Vol 4, 2000 Aug; 43(2 Pt 1):244-8.

Andre J, Achten G. Onychomycosis. Int $J$ Dermatol., 1987; 26: 481-490.

Boni e, Elewski, Onychomycosis: Pathogenesis, Diagnosis, and Management, Clinical, Microbiol, Rev. July 1998: 11(3):415429.

Bramono. The Asian Achilles survey, Presented in the $6^{\text {th }}$ Asian Dermatological Congress, Bangkok: November 2001

Bronson D M, D R Desai, S Barskey and S McMillen Foley. An epidemic of infection with Trichophyton tonsurans revealed in a 20 year survey of fungal infections in Chicago. $J$ Am Acad Dermatol., 1983; 8: 322-330

Charif M A, Elewski B E. A Historical perspective on Onychomycosis. Dermatol Ther., 1997: 3: 43-45
Cohen J L, Scher R K, Papper A S. The nail and fungal infections. Cutaneous fungal infections, New York, NY: Igaku-Shoin Inc; 1992. Pp. 106-122

Dompmartin D, Dompmartin A, Deluol A M, Grosshans E, Coulaud J P. Onychomycosis and AIDS: Clinical and laboratory findings in 62 patients. Int $J$ Dermatol 1990; 29: 337-339.

H. Heikkila, S. Stubb, The prevalence of onychomycosis in Finland, vol 133, issue 5, November 1995, page 699-703.

Jagdish Chander, Text Book of Medical Mycology, $4^{\text {th }}$ Edition (2107).

Kaur R, Kasyap B, Bhalla P. A five year survey of onychomycosis in New Delhi, India: Epidemiological and Laboratory aspects. Indian J Dermatol 2007;52:39-42

Mugge, Haustein UF, Nenoff P. Causative agents of onychomycosis- A retrospective study. J Dtsch Dermatol Ges.2006 Mar;4(3):218-28

Ramudamu, Mandira, W Lyngdoh, Valarie; Prasad, Abhijit; Rajbongshi, Jyotismita; Durairaj, Elantamilan. (2018). A Study on the Mycological Profile of Onychomycosis in a Tertiary Care Hospital in Northeast India. Indian Journal of Applied research. 8. 306-9.

Sigurgeirsson B, Baran R. Prevalence of onychomycosis in the global populationA literature Study. Journal of European Academy of Dermatology and Venereology2014 Nov; 28(11): 1480-91.

Zaias N, Tosti A, Rebell G, Morelli R, Bardazzi F, Bieley H, Zaiac N, Glick B, Paley B, Allevato M, Baran R. Autosomal dominant pattern of Distal subungual onychomycosis caused by Trichophyton rubrum. J Am Acad Dermatol 1996; 34: 302-304.

Zaias N. Onychomycosis. Arch Dermatol 1972; 105: 263-274

\section{How to cite this article:}

Vinay Hajare, G.P. Aaftab and Abdul Hadi Waseem. 2018. A Prospective Study on the Epidemiology of Onychomycosis in Tertiary Care Hospital. Int.J.Curr.Microbiol.App.Sci. 7(08): 3765-3770. doi: https://doi.org/10.20546/ijcmas.2018.708.383 